Journal of Organometallic Chemistry, 252 (1983) 187-194

Elsevier Sequoia S.A., Lausanne - Printed in The Netherlands

\title{
METALLORGANISCHE LEWIS-SÄUREN; METALLKOMPLEXE MIT SCHWACH KOORDINIERTEN ANIONISCHEN LIGANDEN
}

\section{XIII *. NACHWEIS DER KOORDINATION VON TETRAFLUOROBORAT IN CARBONYLMOLYBDÄN- UND -WOLFRAM-KOMPLEXEN DURCH ${ }^{19} \mathrm{~F}$ - UND ${ }^{31}$ P-NMR-SPEKTROSKOPIE}

\author{
KARLHEINZ SÜNKEL, GÜNTER URBAN und WOLFGANG BECK * \\ Institut für Anorganische Chemie der Universität München, D-8000 München 2, Meiserstr. I (B.R.D.) \\ (Eingegangen den 10.Februar 1983)
}

\section{Summary}

In the tetrafluoroborato complexes $\left(\eta^{5}-\mathrm{C}_{5} \mathrm{H}_{5}\right)(\mathrm{CO})_{2} \mathrm{LMFBF}_{3}(\mathrm{M}=\mathrm{Mo}, \mathrm{W} ; \mathrm{L}=$ $\left.\mathrm{CO}, \mathrm{PPh}_{3}, \mathrm{P}(\mathrm{OPh})_{3}\right)$ and $\left(\eta^{5}-\mathrm{C}_{9} \mathrm{H}_{7}\right)(\mathrm{CO})_{3} \mathrm{WFBF}_{3}$ the coordinated fluorine atom and the terminal $\mathrm{F}$ atoms of the $\mathrm{BF}_{4}$ ligand can be distinguished by their ${ }^{19} \mathrm{~F}$ NMR signals. ${ }^{19} \mathrm{~F}$ and ${ }^{31} \mathrm{P}$ NMR spectra of $\left(\eta^{5}-\mathrm{C}_{5} \mathrm{H}_{5}\right)(\mathrm{CO})_{2} \mathrm{P}(\mathrm{OPh})_{3} \mathrm{WFBF}_{3}$ allow to establish cis $\rightarrow$ trans isomerization at clevated temperatures as well as rapid rotation of the coordinated $\mathrm{BF}_{4}$ ligand.

\section{Zusammenfassung}

In den Tetrafluoroborato-Komplexen $\left(\eta^{5}-\mathrm{C}_{5} \mathrm{H}_{5}\right)(\mathrm{CO})_{2} \mathrm{LMFBF}_{3}(\mathrm{M}=\mathrm{Mo}, \mathrm{W}$; $\left.\mathrm{L}=\mathrm{CO}, \mathrm{PPh}_{3}, \mathrm{P}(\mathrm{OPh})_{3}\right)$ und $\left(\eta^{5}-\mathrm{C}_{9} \mathrm{H}_{7}\right)(\mathrm{CO})_{3} \mathrm{WFBF}_{3}$ lassen sich das koordinierte Fluoratom und die endständigen F-Atome des $\mathrm{BF}_{4}$-Liganden durch ihre ${ }^{19} \mathrm{~F}$-NMRSignale unterscheiden. An Hand der ${ }^{19} \mathrm{~F}$ - und ${ }^{31} \mathrm{P}$-NMR-Spektren lässt sich für $\left(\eta^{5}-\mathrm{C}_{5} \mathrm{H}_{5}\right)(\mathrm{CO})_{2} \mathrm{P}(\mathrm{OPh})_{3} \mathrm{WFBF}_{3}$ eine bei höherer Temperatur erfolgende cis $\rightarrow$ transIsomerisierung sowie eine rasche Rotation des koordinierten $\mathrm{BF}_{4}$-Liganden nachweisen.

\section{Einleitung}

Die Koordination des $\mathrm{BF}_{4}{ }^{-}$-Ions an ein Metallatom $\mathrm{M}\left(\mathrm{M}-F-\mathrm{BF}_{3}\right)$ wurde bisher vor allem durch IR-Spektroskopie [2] und Röntgenstrukturanalyse [3] nachgewiesen. Von Fischer et al. [4] und Kawaguchi et al. [5] wurden einige TetrafluoroboratoKomplexe NMR-spektroskopisch untersucht.

* XII. Mitteilung siehe Ref. 1 . 
Im ${ }^{13} \mathrm{C}$-NMR-Spektrum von mer- $(\mathrm{MeC}) \mathrm{Cr}(\mathrm{CO})_{3}\left(\mathrm{PMe}_{3}\right) \mathrm{FBF}_{3}$ [2] erscheint das Kohlenstoff-(Carbin)-Signal nicht als Dublett, sondern als Multiplett, was als Beweis für die Koordination des $\mathrm{BF}_{4}{ }^{-}$-Liganden angesehen werden kann. Im ${ }^{31} \mathrm{P}-\mathrm{NMR}$ Spektrum dieser Verbindung wurde kein Dublett sondern ein Quintett gefunden, entsprechend einem raschen Platzwechsel der Fluoratome des koordinierten $\mathrm{BF}_{4}$ Liganden. Das ${ }^{19} \mathrm{~F}$-NMR-Spektrum von trans-W(CO) ${ }_{4}(\mathrm{CPh})\left(\mathrm{FBF}_{3}\right)$ [4] zeigte bei $-50^{\circ} \mathrm{C}$ nur ein breites Singulett bei $\delta-173.5 \mathrm{ppm}$. Für trans- $\mathrm{Pd}\left(\mathrm{FBF}_{3}\right)$ (R) $\left(\mathrm{C}_{5} \mathrm{H}_{5} \mathrm{~N}\right) \mathrm{PPh}_{3}[5]$ wurde im ${ }^{19} \mathrm{~F}-\mathrm{NMR}$-Spektrum ein Doppeldublett bei etwa $-155 \mathrm{ppm}$ und ein eng benachbartes, zu höheren Frequenzen verschobenes Multiplett gefunden. Die wenigen ${ }^{19} \mathrm{~F}-\mathrm{NMR}$-spektroskopisch untersuchten Fluorokomplexe $\mathrm{L}_{n} \mathrm{M}-\mathrm{F}$ von Metallen in niederen Oxidationsstufen zeigen, dass das koordinierte Fluoratom durchweg sehr gut abgeschirmt ist [6,7] (vgl. Tab. 1). Daher sollten sich das koordinierte Fluoratom $\left(\mathrm{M}-F-\mathrm{BF}_{3}\right)$ und die endständigen Fluoratome $\left(-\mathrm{B} F_{3}\right)$ in ihrer chemischen Verschiebung stark unterscheiden und zwat müsste die Resonanz des koordinierten Fluoratoms bei niedrigeren Frequenzen relativ zu $\mathrm{BF}_{4}^{-}$auftreten. Wir haben nun eine Reihe von TetrafluoroboratoKomplexen des Typs $\left(\eta^{5}-\mathrm{C}_{5} \mathrm{H}_{5}\right)(\mathrm{CO})_{2}(\mathrm{~L}) \mathrm{MFBF}_{3}\left(\mathrm{~L}=\mathrm{CO}, \mathrm{PPh}_{3}, \mathrm{P}(\mathrm{OPh})_{3} ; \mathrm{M}=\mathrm{Mo}\right.$, W) $[8,9]{ }^{19} \mathrm{~F}$ - und ${ }^{31} \mathrm{P}$-NMR-spektroskopisch bei tiefen Temperaturen vermessen.

Tetrafluoroborato-Komplexe $\left(\eta^{5}-\mathrm{C}_{5} \mathrm{H}_{5}\right)(\mathrm{CO})_{2} \mathrm{LMFBF}_{3}\left(\mathrm{M}=\mathrm{Mo}, \mathrm{W} ; \mathrm{L}=\mathrm{CO}, \mathrm{PPh}_{3}\right.$, $\left.\mathbf{P}(\mathrm{OPh})_{3}\right)$

Wie erwartet, werden in den ${ }^{19} \mathrm{~F}-\mathrm{NMR}$-Spektren von $\left(\eta^{5}-\mathrm{C}_{5} \mathrm{H}_{5}\right)(\mathrm{CO})_{3} \mathrm{MFBF}_{3}$ $(\mathrm{M}=\mathrm{Mo}, \mathrm{W})$ jeweils ein Dublett $\left(-\mathrm{FB} F_{3}\right)$ bei etwa $-155 \mathrm{ppm}$ und ein Quartett $\left(-\mathrm{FBF}_{3}\right)$ bei -347.6 bis $-417.5 \mathrm{ppm}$ gefunden (im Intensitätsverhältnis $3 / 1$ ) (Tab.1, Fig. 1). Diese grosse Verschiebung des $\mathrm{M}-F-\mathrm{BF}_{3}$-Signals könnte dafür verantwortlich sein, dass bei den bisher ${ }^{19}$ F-NMR-spektroskopisch untersuchten Tetrafluoroborato-Komplexen [4,5] das Signal des koordinierten Fluoratoms nicht erfasst wurde. In den $\mathrm{P}(\mathrm{OPh})_{3}$-Komplexen sind die Signale des koordinierten Fluoratoms durch die Kopplung mit dem ${ }^{31} \mathrm{P}$-Atom in Dubletts aufgespalten. Bei den phosphanhaltigen Komplexen besteht die Möglichkeit der cis-trans Isomerie. Dies führt im Fall von $\left(\eta^{5}-\mathrm{C}_{5} \mathrm{H}_{5}\right)(\mathrm{CO})_{2} \mathrm{P}(\mathrm{OPh})_{3} \mathrm{WFBF}_{3}$ zu je zwei unabhängigen

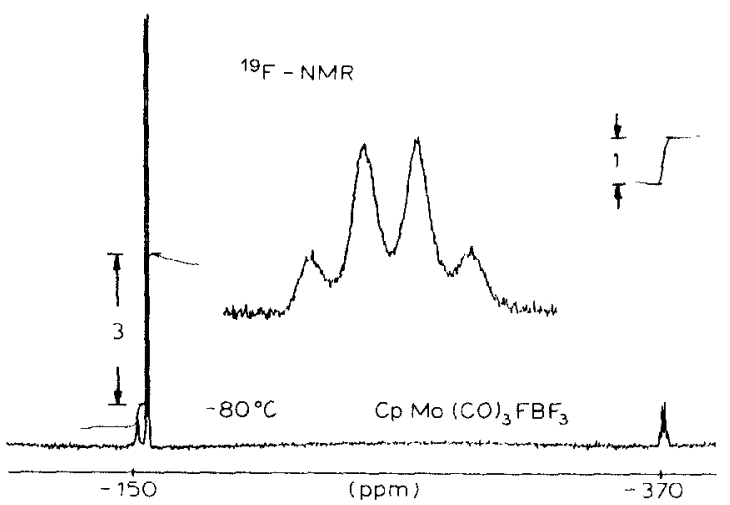

Fig. 1. ${ }^{19} \mathrm{~F}-\mathrm{NMR}$-Spektrum von $\left(\eta^{5}-\mathrm{C}_{5} \mathrm{H}_{5}\right)(\mathrm{CO})_{3} \mathrm{MoFBF}_{3}$ in $\mathrm{CD}_{2} \mathrm{Cl}_{2}$ bei $-80^{\circ} \mathrm{C}$. 


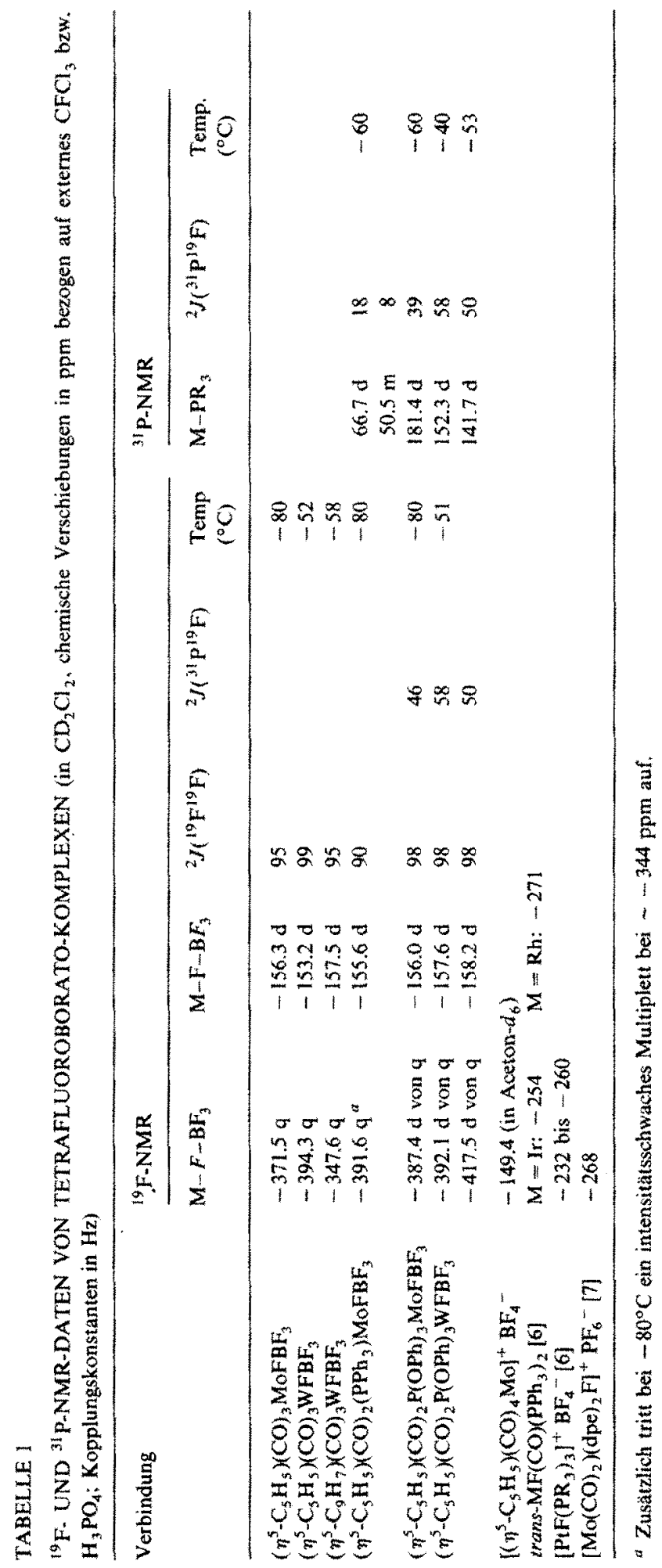


Dubletts und Oktetts (siehe unten). Für den Indenylkomplex $\left(\eta^{5}-\mathrm{C}_{9} \mathrm{H}_{7}\right)(\mathrm{CO})_{3} \mathrm{WFBF}_{3}$, der im IR-Spektrum die stärkste Aufspaltung der $\nu(B-F)$-Banden zeigt [8], wird von den vermessenen Komplexen für das koordinierte Fluoratom die geringste $\mathrm{Ab}$ schirmung beobachtet. Es lässt sich offenbar keine Korrelation zwischen der Aufspaltung der $\nu(B-F)$-Banden im IR-Spektrum und der ${ }^{19} \mathrm{~F}$ chemischen Verschiebung aufstellen.

Bei Temperaturerhöhung ist allgemein eine Intensitätsabnahme und gleichzeitige Verbreiterung der Fluorsignale zu beobachten. Bei keiner der untersuchten Verbindungen liess sich im untersuchten Temperaturbereich ein rotierender, aber noch koordinierter $\mathrm{BF}_{4}$-Ligand ${ }^{19} \mathrm{~F}-\mathrm{NMR}$-spektroskopisch nachweisen. Dies gelang allerdings durch ${ }^{31}$ P-NMR-Spektroskopie. Bei tiefen Temperaturen sind die ${ }^{31} \mathrm{P}$-Signale zu Dubletts aufgespalten, die sich bei Temperaturerhöhung verbreitern und schliesslich eine quintettartige Struktur annehmen. Die gemittelte Kopplungskonstante ${ }^{2} J\left({ }^{31} \mathrm{P}^{19} \mathrm{~F}\right)$ beträgt - entsprechend vier äquivalenten F-Atomen - etwa ein Viertel der Kopplungskonstante im Dublett. Letzteres bestätigt die Untersuchungen von E.O. Fischer und Mitarb. an $\mathrm{Cr}(\mathrm{CO})_{3}\left(\mathrm{PMe}_{3}\right)(\mathrm{CMe})\left(\mathrm{BF}_{4}\right)$ [2]. Warum das bei Rotation des $\mathrm{BF}_{4}{ }^{-}$zu erwartende Dublett im ${ }^{19}$ F-NMR-Spektrum nicht beobachtet werden konnte, bleibt vorläufig unklar. Möglicherweise ist die scalare Relaxation 2. Art durch B-F-Kopplung dafür verantwortlich.

\section{cis-trans-Isomerisierung von $\left(\eta^{5}-\mathrm{C}_{5} \mathrm{H}_{5}\right)(\mathrm{CO})_{2} \mathrm{P}(\mathrm{OPh})_{3} \mathrm{WFBF}_{3}$}

Die Verbindungen des Typs $\left(\eta^{5}-\mathrm{C}_{5} \mathrm{H}_{5}\right) \mathrm{M}(\mathrm{CO})_{2} \mathrm{LX}$ haben annähernd quadratisch pyramidale Struktur und können deshalb als cis-trans-Isomere vorliegen. Nach Untersuchungen von Faller et al. [10] wird das Gleichgewicht zwischen den Isomeren sowohl von sterischen als auch elektronischen Effekten beeinflusst.

Die Hydrido-Komplexe $(\mathrm{X}=\mathrm{H})$ liegen meist bevorzugt in der cis-Konfiguration vor $[10]$, mit $\mathrm{L}=\mathrm{P}(\mathrm{OPh})_{3}$ sogar fast vollständig. Der Übergang zu sterisch anspruchsvolleren Liganden $\mathrm{X}$ wie z.B. $\mathrm{I}^{-}, \mathrm{CH}_{3}$ oder $\mathrm{CH}_{2} \mathrm{Ph}$ führt bevorzugt zum trans-Isomeren.

In der Reaktion von $\left(\eta^{5}-\mathrm{C}_{5} \mathrm{H}_{5}\right)(\mathrm{CO})_{2} \mathrm{P}(\mathrm{OPh})_{3} \mathrm{~W}-\mathrm{H}$ mit $\mathrm{Ph}_{3} \mathrm{C}^{+} \mathrm{BF}_{4}^{-}$zu $\left(\eta^{5}-\right.$ $\left.\mathrm{C}_{5} \mathrm{H}_{5}\right)(\mathrm{CO})_{2} \mathrm{P}(\mathrm{OPh})_{3} \mathrm{WFBF}_{3}$ sollte also, sofern nur sterische Effekte massgebend sind, aus dem reinen cis-Hydrid vor allem der trans-Tetrafluoroborato-Komplex entstehen. Wie Fig. 2 und 3 zeigen, ist eine solche Umwandlung auch tatsächlich in den ${ }^{19} \mathrm{~F}$ - und ${ }^{31} \mathrm{P}$-NMR-Spektren zu beobachten. Geht man davon aus, dass das $\mathrm{BF}_{4}{ }^{-}$-Ion den Platz des Hydridliganden in der Koordinationssphäre des Wolframs einnimmt, so sollte bei tiefer Temperatur zunächst der cis-Tetrafluoroborato-Komplex entstehen. Bei der Reaktion von cis- $\left(\eta^{5}-\mathrm{C}_{5} \mathrm{H}_{5}\right)(\mathrm{CO})_{2} \mathrm{LMoH}\left(\mathrm{L}=\mathrm{PPh}_{3}\right.$, $\left.\mathrm{P}(\mathrm{OPh})_{3}\right)$ mit $\mathrm{CCl}_{4}$ zu cis- $\left(\eta^{5}-\mathrm{C}_{5} \mathrm{H}_{5}\right)(\mathrm{CO})_{2} \mathrm{LMoCl}$ wird ebenfalls Konfigurationserhalt gefunden [11].

Das ${ }^{31}$ P-NMR-Spektrum zeigt zunächst nur ein Dublett. Bei etwa $+10^{\circ} \mathrm{C}$ erscheint neben dem Quintett, das aus dem Dublett hervorgegangen ist, ein weiteres zu höheren Frequenzen verschobenes Quintett, das schliesslich bei dieser Temperatur allein übrigbleibt. Bei erneutem Abkühlen wandelt sich dieses Quintett wieder in ein Dublett um, das ursprüngliche Dublett bildet sich nicht mehr zurück. Im IRSpektrum lässt sich aus der relativen Intensität der Carbonylbanden der so erhaltenen Lösung auf trans-Konfiguration des Komplexes schliessen [12].

Dies bestätigt die Annahme, dass ursprünglich ein cis-Komplex gebildet wurde, 


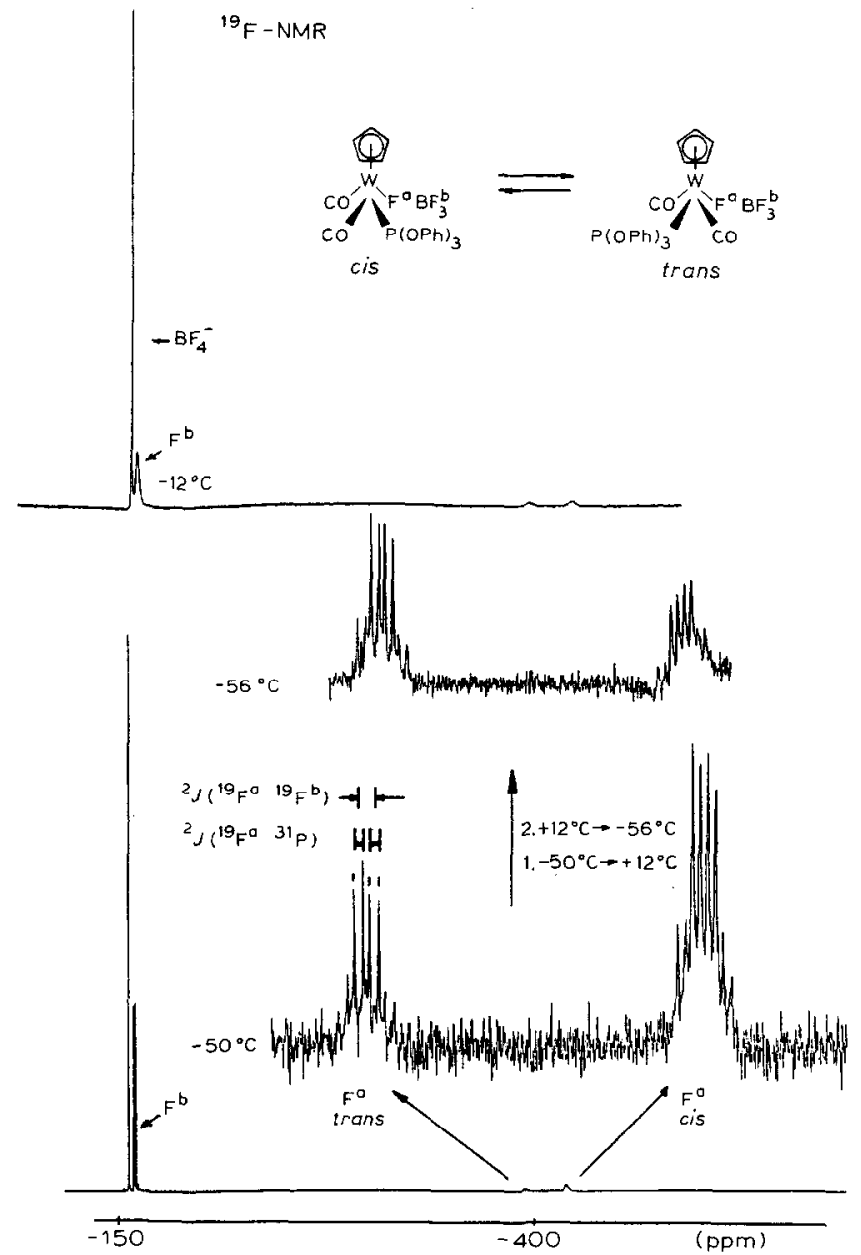

Fig. 2. ${ }^{19} \mathrm{~F}-\mathrm{NMR}-\mathrm{Spektren}$ von $\left(\eta^{5}-\mathrm{C}_{5} \mathrm{H}_{5}\right)(\mathrm{CO})_{2} \mathrm{P}(\mathrm{OPh})_{3} \mathrm{WFBF}_{3}$ zwischen -56 und $+12^{\circ} \mathrm{C}$ in $\mathrm{CD}_{2} \mathrm{Cl}_{2}$.

der sich aus sterischen Erfordernissen in das trans-Isomere umlagert. Das ${ }^{19} \mathrm{~F}$ NMR-Spektrum von $\left(\eta^{5}-\mathrm{C}_{5} \mathrm{H}_{5}\right)(\mathrm{CO})_{2} \mathrm{P}(\mathrm{OPh})_{3} \mathrm{WFBF}_{3}$ (Fig. 2) zeigt im Prinzip die gleiche Tendenz; es werden jedoch sofort beide Isomeren nebeneinander beobachtet. Eine Zuordnung der Isomeren aufgrund der relativen chemischen Verschiebung oder der Kopplungskonstante allein erscheint schwierig, da zu wenige Vergleichsverbindungen vorliegen. In quadratisch planaren Komplexen liegen die cis ${ }^{2} J\left({ }^{31} \mathbf{P}^{19} \mathbf{F}\right)$ Kopplungskonstanten bei $28-39 \mathrm{~Hz}$, die entsprechenden trans-Kopplungen sind meist wesentlich grösser $(140-160 \mathrm{~Hz})[6]$. Ähnliche Beobachtungen gelten für die ${ }^{2} J\left({ }^{31} \mathrm{P}^{1} \mathrm{H}\right)$-Kopplungen in quadratisch-planaren Hydrido-Komplexen [13]. Dagegen ist für quadratisch pyramidale Hydrido-Komplexe des Typs $\left(\eta^{5}-\mathrm{C}_{5} \mathrm{H}_{5}\right)(\mathrm{CO})_{2^{-}}$ $\left(\mathrm{PR}_{3}\right) \mathrm{MH}$ die ${ }^{2} J\left({ }^{31} \mathrm{P}^{\mathrm{l}} \mathrm{H}\right)$-Kopplungskonstante im cis-Isomeren etwa zwei- bis dreimal grösser als im trans-Isomeren [13,14]. 


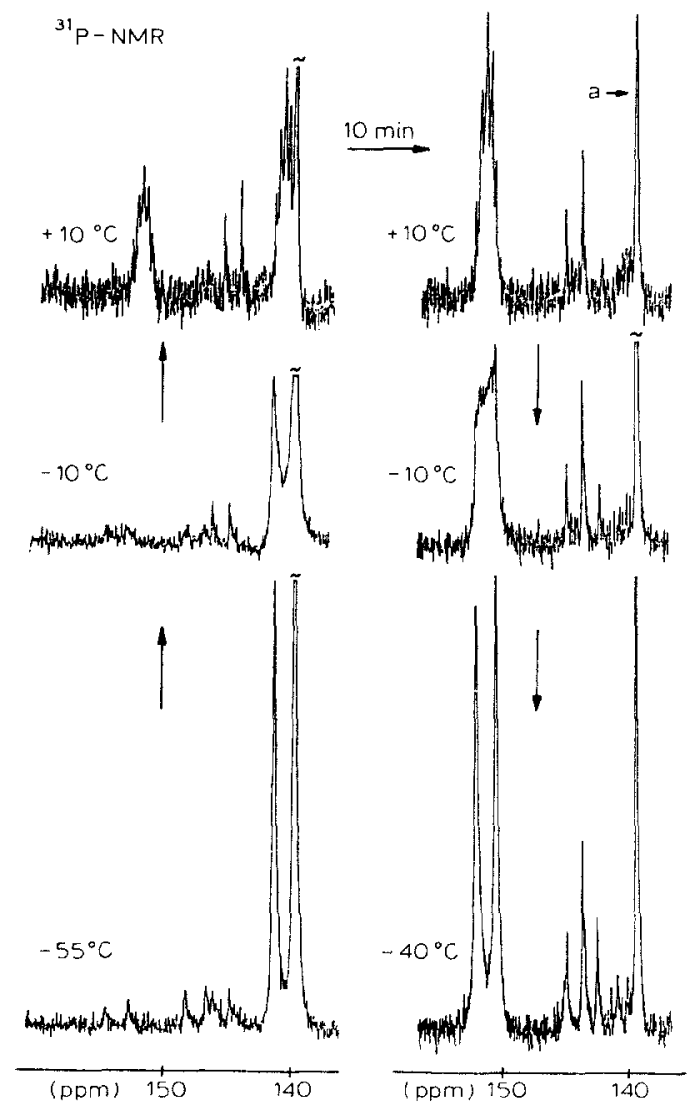

Fig. 3. ${ }^{3 !} \mathrm{P}-\mathrm{NMR}$-Spektren von $\left(\eta^{5}-\mathrm{C}_{5} \mathrm{H}_{5}\right)(\mathrm{CO})_{2} \mathrm{P}(\mathrm{OPh})_{3} \mathrm{WFBF}_{3}$ bei verschiedenen Temperaturen in $\mathrm{CD}_{2} \mathrm{Cl}_{2} ; \mathrm{a}=$ Zersetzungsprodukt.

NMR-spektroskopischer Hinweis auf einen Methylenchlorid-Komplex, I( $\left.\eta^{5}-C_{5} H_{5}\right)$ $\left.(\mathrm{CO})_{3} \mathrm{Mo}\left(\mathrm{CH}_{2} \mathrm{Cl}_{2}\right)\right]^{+} \mathrm{PF}_{6}{ }^{-}$

Bei der Darstellung von $\left(\eta^{5}-\mathrm{C}_{5} \mathrm{H}_{5}\right)(\mathrm{CO})_{3} \mathrm{MFEF}_{5}(\mathrm{M}=\mathrm{Mo}, \mathrm{W} ; \mathrm{E}=\mathrm{P}, \mathrm{As}, \mathrm{Sb})$ in $\mathrm{CH}_{2} \mathrm{Cl}_{2}$ fallen im Unterschied zum $\mathrm{BF}_{4}{ }^{-}$-Komplex zunächst purpurfarbene Niederschläge an, die erst nach längerem Trocknen bei Raumtemperatur die übliche blau-violette Farbe annehmen [8,1]. Die ${ }^{1} \mathrm{H}-\mathrm{NMR}-\mathrm{Spektren}$ und die Analysen dieser purpurfarbenen Niederschläge zeigen, dass sie $\mathrm{CH}_{2} \mathrm{Cl}_{2}$ enthalten. Im IR-Spektrum können zwei $\nu(\mathrm{C}-\mathrm{Cl})$-Banden koordiniertem $\mathrm{CH}_{2} \mathrm{Cl}_{2}$ zugeordnet werden.

Metallkomplexe mil halogenierten Kohlenwasserstoffen als Liganden wurden schon öfters diskutiert [15]. Vor kurzem wurde die Bindung von o-Diiodbenzol an Iridium(III) röntgenographisch nachgewiesen [16]. Das ${ }^{19} \mathrm{~F}-\mathrm{NMR}-\mathrm{Spektrum}$ von $\left(\eta^{5}-\mathrm{C}_{5} \mathrm{H}_{5}\right)(\mathrm{CO})_{3} \mathrm{MoPF}_{6}$ in $\mathrm{CD}_{2} \mathrm{Cl}_{2}$ zeigt bei Temperaturen unter $-20^{\circ} \mathrm{C}$ nur ein Dublett bei $-70.5 \mathrm{ppm},{ }^{1} J\left({ }^{31} \mathrm{P}^{19} \mathrm{~F}\right) 714 \mathrm{~Hz}$, das wir freiem, unkoordinierten $\mathrm{PF}_{6}{ }^{-}$ zuordnen. Erhöht man die Temperatur bis auf $+15^{\circ} \mathrm{C}$, so nimmt die Intensität des Dubletts des freien $\mathrm{PF}_{6}{ }^{-} \mathrm{ab}$. Gleichzeitig wachsen bei niederen Frequenzen 4 kleine intensitätsschwache Resonanzsignale $\left(T-10^{\circ} \mathrm{C}:-81 ;-84.3 ;-92.5 ;-97 \mathrm{ppm}\right)$ 
und ein sehr breites, intensitätsstarkes Dublett $\left(T=-10^{\circ} \mathrm{C} ;-111.3 \mathrm{ppm}\right.$; $\left.{ }^{1} J\left({ }^{31} \mathrm{P}^{19} \mathrm{~F}\right) \approx 710 \mathrm{~Hz}\right)$ heraus. Bis etwa $-600 \mathrm{ppm}$ ist kein weiteres Signal zu beobachten. Für koordiniertes Hexafluorophosphat ist ein Spektrum höherer Ordnung zu erwarten [17-19].

Wird die Lösung wieder abgekühlt, so bleibt nur noch das ursprüngliche Dublett erhalten. Diese Beobachtung kann so erklärt werden, dass bei tiefer Temperatur ein $\mathrm{CH}_{2} \mathrm{Cl}_{2}-\mathrm{K}$ omplex vorliegt, der freies unkoordiniertes $\mathrm{PF}_{6}{ }^{-}$enthält und somit zu dem Dublett-Signal führt. Bei Temperaturerhöhung verdrängt das $\mathrm{PF}_{6}{ }^{-}$-Ion allmählich das $\mathrm{CH}_{2} \mathrm{Cl}_{2}$-Molekül und tritt in die Koordinationsphäre des Molybdäns ein.

$$
\left[\left(\eta^{5}-\mathrm{C}_{5} \mathrm{H}_{5}\right)(\mathrm{CO})_{3} \mathrm{Mo}\left(\mathrm{CH}_{2} \mathrm{Cl}_{2}\right)\right]^{+} \mathrm{PF}_{6}-\stackrel{\Delta}{\rightleftarrows}\left(\eta^{5}-\mathrm{C}_{5} \mathrm{H}_{5}\right)(\mathrm{CO})_{3} \mathrm{MoFPF}_{5}+\mathrm{CH}_{2} \mathrm{Cl}_{2}
$$

Bei höherer Temperatur besteht die Möglichkeit der Rotation des koordinierten $\mathrm{PF}_{6}{ }^{-}$-Ions. Dies ist offensichtlich bei den in der I.iteratur beschriebenen $\mathrm{PF}_{6}{ }^{-}$Komplexen der Fall, die durchwegs nur zu Dubletts bei -72 bis -75 ppm und Kopplungskonstanten von etwa $750 \mathrm{~Hz}$ Anlass geben [20].

Im ${ }^{1} \mathrm{H}$-NMR Spektrum konnten bei -60 bis $0^{\circ} \mathrm{C}$ keine 2 Signale für koordiniertes, bzw. unkoordiniertes $\mathrm{CH}_{2} \mathrm{Cl}_{2}$ gefunden werden. Möglicherweise ist die chemische Verschiebung zu klein, so dass die Signale zusammenfallen.

Ausserdem wird die Intensität des $\left(\eta^{5}-\mathrm{C}_{5} \mathrm{H}_{5}\right)$-Signals $\left(6.04 \mathrm{ppm}\right.$ rel. $\mathrm{CHDCl}_{2} 5.30$ ppm) bei zunehmender Temperatur geringer, was einer abnehmenden Löslichkeit entspricht. Kühlt man wieder ab, so erscheint das $\left(\eta^{5}-\mathrm{C}_{5} \mathrm{H}_{5}\right)$-Signal fast in seiner ursprünglichen Intensität wieder.

Bei allen untersuchten Komplexen $\left(\eta^{5}-\mathrm{C}_{5} \mathrm{H}_{5}\right)(\mathrm{CO})_{2} \mathrm{LMX}(\mathrm{M}=\mathrm{Mo}, \mathrm{W} ; \mathrm{L}=\mathrm{CO}$, $\left.\mathrm{PPh}_{3}, \mathrm{P}(\mathrm{OPh})_{3} ; \mathrm{X}=\mathrm{BF}_{4}^{-}, \mathrm{PF}_{6}{ }^{-}\right)$treten in den NMR-Spektren bei höheren Temperaturen allmählich die Signale der freien Anionen auf $\left(\mathrm{BF}_{4}{ }^{-}: \delta\left({ }^{19} \mathrm{~F}\right)-149\right.$ bis 154 ppm; $\left.\mathrm{PF}_{6}{ }^{-}: \delta\left({ }^{19} \mathrm{~F}\right)-72 \mathrm{ppm}\right)$. Dies ist auf die Bildung der stabilen 18-Elektronensysteme $\left[\left(\eta^{5}-\mathrm{C}_{5} \mathrm{H}_{5}\right)(\mathrm{CO})_{4} \mathrm{M}\right]^{+} \mathrm{X}^{-}, \quad\left[\left(\eta^{5}-\mathrm{C}_{5} \mathrm{H}_{5}\right)(\mathrm{CO})_{3} \mathrm{LM}\right]^{+} \mathrm{X}^{-}$bzw. $\left[\left(\eta^{5}-\right.\right.$ $\left.\left.\mathrm{C}_{5} \mathrm{H}_{5}\right)(\mathrm{CO})_{2} \mathrm{~L}_{2} \mathrm{M}\right]^{+} \mathrm{X}^{-}[8,9]$ zurückzuführen, die durch Ligandenübertragung entstehen.

\section{Experimentelles}

Die untersuchten Komplexe wurden, wie früher beschrieben, dargestellt $[8,9]$. Für die Messungen stand ein Jeol FX-90-NMR-Gerät zur Verfügung.

\section{Dank}

Der Deutschen Forschungsgemeinschaft und dem Fonds der Chemischen Industrie gilt unser Dank für grosszügige Förderung. Für wertvolle Anregungen bei der Diskussion der NMR-Spektren danken wir Herrn Dr. B. Wrackmeyer sowie Frl. U. Taubald, Frl. E. Eichin und Frl. D. Motz für aktive Mitarbeit.

\section{Literatur}

1 K. Sünkel, U. Nagel und W. Beck, J. Organomet. Chem., 251 (1983) 227.

2 K. Richter, E.O. Fischer und C.O. Kreiter, J. Organomet. Chem., 122 (1976) 187.

3 B. Olgemöller, H. Bauer, H. Löbermann, U. Nagel und W. Beck, Chem. Ber., 115 (1982) 2271.

4 E.O. Fischer, S. Walz, A. Ruhs und F.R. Kreissl, Chem. Ber., 111 (1978) 2765. 
5 K. Isobe, K. Nanjo, Y. Nakamura und S. Kawaguchi, Chem. Lett., (1979) 1193.

6 M.A. Cairns, K.R. Dixon und J.J. McFarland, J. Chem. Soc., Dalton Trans., (1975) 1159.

7 M.R. Snow und F.L. Wimmer, Aust. J. Chem., 29 (1976) 2349.

8 W. Beck und K. Schloter, Z. Naturforsch. B, 33 (1978) 1214.

9 K. Sünkel, H. Ernst und W. Beck, Z. Naturforsch. B, 36 (1981) 474.

10 J.W. Faller und A.S. Anderson, J. Amer. Chem. Soc., 92 (1970) 5852.

II A. Bainbridge, P.J. Craig und M. Green, J. Chem. Soc. A, (1968) 2715.

12 W. Beck, A. Melnikoff und R. Stahl, Chem. Ber., 99 (1966) 3721.

13 H.D. Kaesz und R.B. Saillant, Chem. Rev., 72 (1972) 231.

14 T.C. Flood, E. Rosenberg und A. Sarhangi, J. Amer. Chem. Soc., 99 (1977) 4334.

15 J.A. Osborn, F.H. Jardine, J.F. Young und G. Wilkinson, J. Chem. Soc. A. (1966) 1711 1; D.N. Lawson, J.A. Osborn und G. Wilkinson, J. Chem. Soc. A, (1966) 1733; A.W. Addison und R.D. Gillard, J. Chem. Soc., Dalton Trans., (1973) 2002; P.J. Harris, S.A.R. Knox, R.J. McKinney und F.G.A. Stone, J. Chem. Soc., Dalton Trans., (1978) 1009.

16 R.H. Crabtree, J.W. Faller, M.F. Mellea und J.M. Quirk, Organometallics, 1 (1982) 1361.

17 J.-Y. Calves und R.J. Gillespie, J. Chem. Soc. Chem. Commun.. (1976) 506.

18 S. Brownstein, J. Bornais und G. Latremouille, Can. J. Chem., 56 (1978) 1419.

19 R.K. Harris und K.J. Packer, J. Chem. Soc., (1962) 3077.

20 Vergl. F.J. Regina und A. Wojcicki, Inorg. Chem., 19 (1980) 3803. 